INFLAMMATION AND INFLAMMATORY BOWEL DISEASE

\title{
Non-steroidal anti-inflammatory drugs as a risk factor for acute diarrhoea: a case crossover study
}

\author{
I Etienney, L Beaugerie, C Viboud, A Flahault
}

Gut $2003 ; 52: 260-263$

See end of article for authors' affiliations

Correspondence to $\operatorname{Pr} L$ Beaugerie, Service de Gastroentérologie, Hôpital Rothschild, 33 Boulevard de Picpus, 75571 Paris

Cedex 12, France:

laurent.beaugerie@

rth.ap-hop-paris.fr

Accepted for publication 15 May 2002

\begin{abstract}
Background and aim: Several cases of acute colitis induced by non-steroidal anti-inflammatory drugs (NSAIDs) have been reported but the general role of recent NSAID intake as a risk factor for acute diarrhoea has not been studied to date. The aim of our study was to determine whether the risk of acute diarrhoea is increased by NSAIDs in a prospective series of acute diarrhoea cases which were seen by general practitioners in France and were serious enough to require a stool culture.

Patients, physicians, and methods: A total of 285 consecutive patients with acute diarrhoea, seen by Sentinel general practitioners (GPs) between December 1998 and July 1999, were enrolled in a case crossover study in which each case served as his/her own control. GPs collected information on exposure to NSAIDs during the four month period preceding the onset of diarrhoea. The relative risk of NSAID related acute diarrhoea was estimated by comparing exposure to NSAIDs during a risk period preceding the onset of diarrhoea with exposure during the first part of the four month observation period. Three risk periods lasting for one, three, and six days before the onset of diarrhoea were considered.

Results: The relative risks of acute diarrhoea due to recent NSAID intake were increased for all three risk periods. These risks and their confidence intervals were $2.9(1.4-6.1)$ for the one day risk period, $2.7(1.4-5.1)$ for the three day period, and $3.3(2.0-5.4)$ for the six day period.

Conclusion: Recent NSAID intake emerges as a risk factor for acute diarrhoea. We suggest that acute diarrhoea seen in general practice, and not only acute colitis seen by gastroenterologists, should be considered as a potential complication of recent NSAID intake.
\end{abstract}

$\mathrm{N}$ on-steroidal anti-inflammatory drugs (NSAIDs) are among the most widely prescribed drugs worldwide and can induce lesions of the entire gastrointestinal tract. The ulcerogenic effects of NSAIDs on the upper gastrointestinal tract are well established ${ }^{1}$ but it is only recently that the small intestinal and colonic lesions induced by these drugs have received closer attention. ${ }^{23}$ NSAIDs can induce small bowel enteropathy with a high prevalence (20-65\% of patients taking these drugs) depending on the method used for diagnosis. ${ }^{4-6}$ This enteropathy often leads to iron deficiency and hypoalbuminaemia but is generally not associated with diarrhoea. ${ }^{7}$ Several cases of acute NSAID induced colitis have been reported. ${ }^{8}$ These de novo colitis cases are usually considered rare but serious and sometimes fatal complications of NSAID therapy. ${ }^{8}{ }^{9}$ However, the case for NSAID induced colitis might be more extensive, as suggested by Glesson and colleagues. ${ }^{10}$ Recent and current use of NSAIDs has been shown to increase the risk of ischaemic colitis and of the first attack or reactivated inflammatory bowel disease. ${ }^{11}{ }^{12}$ In contrast, whether chronic use of NSAIDs is a possible cause of collagenous colitis remains questionable. ${ }^{13}$ All of these lesions have been described in patients with diarrhoea; conversely, preliminary data suggest that chronic use of NSAIDs does not alter histologically colonic mucosa of patients without diarrhoea. ${ }^{15}$ As far as we know, the question of whether NSAID intake can cause acute diarrhoea, irrespective of the presence of underlying lesions, has not been studied to date. The only available data concern the incidence of diarrhoea following the use of meclofenamate sodium, which was estimated as up to $30 \%$ in clinical trials. ${ }^{16}$ The incidence of diarrhoea due to the use of other NSAIDs has been reported to be no greater than $1 \%$ and not significantly different from placebo in controlled clinical trials. ${ }^{16}$

The aim of our case crossover study was to determine whether the risk of acute diarrhoea is increased by current intake of NSAIDs in a prospective series of cases of severe acute diarrhoea.

\section{MATERIAL AND METHODS \\ Material}

Acute diarrhoea is one of the communicable diseases subject to electronic surveillance by France's Sentinel network of general practitioners (GPs). ${ }^{17}$ According to the network's data, approximately three million people in France consult a doctor for acute diarrhoea each year. ${ }^{18}$ Visible blood in stools is reported with a frequency of approximately $1 \%$, and stool examination is performed in less than $5 \%$ of cases in general practice. $^{19}$

We contacted 1000 Sentinel GPs belonging to the French communicable disease network. ${ }^{14}$ They took part in the study on a voluntary unpaid basis, as they do for epidemiological ongoing surveillance. The population studied was selected among the patients of participating Sentinel practitioners and comprised consecutive cases of acute diarrhoea which had lasted for one month and were serious enough to require stool examination

\section{Data collection and statistical analysis}

GPs interviewed patients during consultations. Data were collected concerning patient age and sex, and the following clinical characteristics: date of onset of diarrhoea, fever or dysenteric syndrome, and results of stool examination. Medical history concerned exposure to NSAIDs during the four months preceding the consultation. The type of drug, date of

Abbreviations: NSAIDs, non-steroidal anti-inflammatory drugs; GPs, general practitioners; RR, relative risk. 

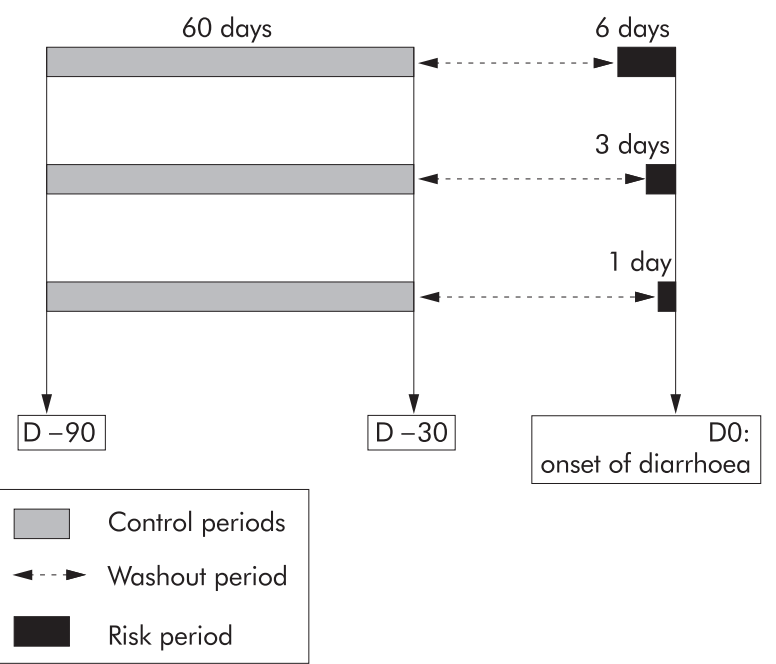

Figure 1 Definition and duration of the control, risk, and washout periods for each duration of risk period (six days, three days, and one day) in the case crossover design.

onset, and duration of each treatment were recorded. When available, information on NSAID intake was totally or partially completed from medical case records.

Standard data quality verification procedures recording data entry checks, code, and programming checks were applied to the data used for analysis. Statistical analysis and estimation of relative risks (RRs) and their confidence intervals were performed using SAS software (version 6; SAS Institute, Cary, North Carolina, USA).

\section{The case crossover design}

Developed by Maclure in 1991, the case crossover design provides a means of studying the effects of transient exposure on the risk of acute outcome. ${ }^{20}$ Only cases are required by this design, as each case acts as its own control. The case crossover design is useful for assessing the effects of exposures just before outcome, as opposed to prior exposures. In this design, the period just before the outcome is termed the risk period. Earlier periods of proportional duration are termed control periods. The time between the risk and control periods is the washout period. In our study, three risk periods of one, three, and six days prior to the onset of diarrhoea were considered. We compared exposures between the one day risk period (respectively three and six day) and 60 control periods (respectively 20 and 10) chosen in the first two months of the patient's drug history (see fig 1 ).

The RR of acute diarrhoea was estimated by comparing exposure to NSAIDs reported in the risk period with exposure reported in the control periods, as described by Maclure. ${ }^{20}$ Two variables $\mathrm{X}$ and $\mathrm{P}$ were defined for each subject, and each subject contributed to the numerator and denominator of the RR as follows: let $X=1$ if the subject was exposed in the risk period, else $\mathrm{X}=0$; let $\mathrm{P}$ be the proportion of control periods in which the subject was exposed. The numerator is the sum of $\mathrm{X} \times(\mathrm{l}-\mathrm{P})$ over all cases. The denominator is the sum of $\mathrm{P} \times(1-\mathrm{X})$ over all cases. Confidence intervals were estimated as described by Maclure. ${ }^{20}$ Note that in the case crossover analysis, subjects not exposed in the risk period $(X=0)$ and in the control periods $(\mathrm{P}=0)$, as well as subjects exposed in the risk period $(\mathrm{X}=1)$ and in all control periods $(\mathrm{P}=1)$ do not contribute to the estimation of RR.

\section{RESULTS}

Of the 1000 Sentinel GPs contacted, 930 were available by telephone but 454 did not want to participate in the study, and 269 agreed to participate but did not report any cases. Finally,
Table 1 Demographic and clinical characteristics of 285 patients

\begin{tabular}{ll}
\hline & Distribution \\
\hline Sex (M/F) (n) & $140 / 145$ \\
Age (median-extremes) & 35 y (4 months-89 y) \\
Duration of diarrhoea at consultation (days) & $5(1-30)$ \\
Blood in stools (\% of patients) & 10 \\
Mucus in stools (\% of patients) & 42 \\
Fever (\% of patients) & 44 \\
\hline
\end{tabular}

Table 2 Results of stool examination

\begin{tabular}{ll}
\hline & Infectious agents \\
\hline None & $\mathrm{n}=231$ \\
Bacteria & $\mathrm{n}=41$ \\
& Salmonella (23) \\
& Campylobacter (6) \\
& Escherichia coli pathogen (5) \\
& Shigella (3) \\
& Clostridium difficile (2) \\
& Yersinia (2) \\
& $\mathrm{n}=7$ \\
Parasites & Giardia (5) \\
& Entamoeba histolytica (2) \\
Viruses & $\mathrm{n}=6$ \\
& Adenovirus (3) \\
& Rotavirus (3) \\
\hline
\end{tabular}

285 consecutive patients with acute diarrhoea, seen by 207 Sentinel practitioners between December 1998 and July 1999, were enrolled in the study. Their demographic and clinical characteristics are shown in table 1 . In all cases, diarrhoea was serious enough to require stool examination. Bacterial intestinal pathogens, in most cases Salmonella, were identified in 41 patients $(14.4 \%)$ from routine stool examination (table 2 ). One had recently been exposed to NSAIDs.

The RR of acute diarrhoea due to recent NSAID intake was significantly increased for the three risk periods preceding the onset of the disease. RR values were 2.9 (1.4-6.1) for the one day period, $2.7(1.4-5.1)$ for the three day period, and 3.3 (2.0-5.4) for the six day period (table 3).

\section{DISCUSSION}

Our study using the case crossover design shows firstly that in general practice, recent NSAID intake increases the risk of acute diarrhoea when the disease is severe enough to require stool examination.

This design proved original and effective. ${ }^{21}$ To our knowledge, it has never been used previously in gastroenterology. Secondly, the present work demonstrates that the case crossover design can easily be applied in general medical practice. It was first used to examine the transient effects of brief exposure on the risk of acute events. ${ }^{20}$ This design was subsequently applied to the assessment of various risk factors, including exposure to cellular phones while driving, and physical exertion or anger episodes as potential risk factors of myocardial infarction..$^{21-24}$ Later, the design was used to assess the role of drug exposure or vaccination in various pathologies or adverse events. ${ }^{25-27}$ The case crossover design was shown to be an appropriate alternative to the case control design for assessing rare risks associated with drug exposure. ${ }^{28}$ Chronic use of a medical drug did not modify the magnitude of the estimated RR of an adverse event because data from patients exposed during both risk and control periods were not included in the RR calculation. In addition, no patient was 
Table 3 Exposure to non-steroidal anti-inflammatory drugs (NSAIDs) in the control and risk periods. For each length of the risk periods (one, three, and six days, respectively), the cumulative duration of the control periods was 60 days (see fig 1)

\begin{tabular}{|c|c|c|c|}
\hline & \multicolumn{3}{|c|}{ Length of the risk period } \\
\hline & 1 day & 3 days & 6 days \\
\hline No of control periods & 60 & 20 & 10 \\
\hline Total No of subjects & 285 & 285 & 285 \\
\hline $\begin{array}{l}\text { No of subjects having taken NSAIDs } \\
\text { within the risk period }\end{array}$ & 7 & 8 & 11 \\
\hline $\begin{array}{l}\text { No of subjects having taken NSAIDs at } \\
\text { least once within the control periods }\end{array}$ & 22 & 22 & 22 \\
\hline $\begin{array}{l}\text { Mean proportion of control periods } \\
\text { during which a subject has taken } \\
\text { NSAIDs (min-max) }\end{array}$ & $0.14(0.05-0.43)$ & $0.17(0.05-0.55)$ & $0.24(0.10-0.60)$ \\
\hline $\operatorname{RR}(95 \% \mathrm{Cl})$ & $2.9 \quad(1.4-6.1)$ & $2.7 \quad(1.4-5.1)$ & $3.3 \quad(2.0-5.4)$ \\
\hline
\end{tabular}

$\mathrm{RR}$, relative risk; $\mathrm{Cl}$, confidence interval.

permanently exposed. For mean exposure times of less than one week, and for an acute event as obvious as acute diarrhoea, accurate application of the case control design proved possible in our study. With this design the length of the risk period, or the time lag between the start of exposure and the onset of the event, has to be assumed, if possible from prior knowledge or previous studies. ${ }^{28}$ In a recent review, the duration of exposure to NSAIDs until diagnosis of NSAID induced colitis varied from two days to 12 years, with a median of three months. ${ }^{8}$ Because the pathogenesis of NSAID induced colitis is thought to be due to inhibition of intestinal prostaglandin synthesis during NSAID treatment, ${ }^{8}$ we only took into account recent NSAID intake as a potential triggering factor of diarrhoea.

The case crossover design is also accurate in drug epidemiology, at lower cost than case control studies, as long as the case series is recorded independently of the exposure status. ${ }^{28}$ The case crossover design rules out one of the main causes of bias in case control studies, namely the possible selection of controls who may not be representative of the study population. However, the potential for recall bias was carefully assessed here. A written prescription of NSAIDs during both the control and risk periods was available in our study for $73 \%$ of cases, from medical records. After restricting the analysis to this subgroup, the results did not change substantially (data not shown).

Self medication, unknown to the GP and not noted in the medical record, may also have biased the study. An excess of self medication may have occurred in the risk period, if NSAID intake is a risk factor for acute diarrhoea. This may have led to underestimation of the true level of the risk associated with NSAIDs. Hence this study provides conservative estimates of the risk associated with these drugs. Furthermore, if NSAID intake is a not a risk factor, then self medication will occur to the same extent during the risk and control periods.

As already stated, our study population was selected among cases of acute diarrhoea serious enough to require stool examination. This subgroup represents approximately 5\% of the acute diarrhoea cases seen in general practice. ${ }^{17}$ Although $21(7.4 \%)$ of the 285 patients who were included and had first been seen by GPs were secondarily referred to hospital, this study probably excluded more seriously affected patients who had started treatment in a hospital setting. Clinical diagnosis of severe diarrhoea is based on the presence of marked fever, clinical dehydration, and/or visible blood in stools. Although most of our patients had fever or blood in their stools, an intestinal enteropathogen was only found in $16.8 \%$ of cases. Stool examination was done in general practice, and only for the usual pathogens. Therefore, we cannot exclude the possibility that many of our diarrhoea cases were due to other infectious agents for which stool specimens were not routinely tested.
Diarrhoea secondary to the use of meclofenamate sodium has been reported with a frequency of up to $30 \%$ in clinical trials but none of our patients had taken this drug. Given the diversity of the NSAIDs prescribed (12 different drugs in our study), it was not statistically possible to compare the risks for various types of NSAIDs. The use of NSAIDs may trigger the first attack of inflammatory bowel disease or reactivate it. ${ }^{12}$ We cannot exclude the possibility that some of the patients in our study population may develop overt inflammatory bowel disease during the coming years. However, 250-500 persons per 100000 inhabitants are investigated every year for acute diarrhoea in France but only two cases of inflammatory bowel disease with acute onset are diagnosed. ${ }^{29}{ }^{30}$ The question of the role of NSAIDs in collagenous colitis has been raised but all of our patients had chronic diarrhoea, and the exposure time always exceeded six months. ${ }^{13}$ For these reasons, it is unlikely that any of the cases of acute diarrhoea reported in our study corresponded to collagenous colitis.

The de novo colitis induced by non-steroidal antiinflammatory drugs constitutes a rare but serious and sometimes fatal complication of NSAID intake. ${ }^{8}$ Such cases have been diagnosed in inpatients but not in the setting of general practice. It can be postulated that cases of severe de novo colitis reported represent "the tip of the iceberg" and that milder cases of colitis may not be diagnosed. ${ }^{8}$ Our results suggest that certain cases of severe acute diarrhoea, and not only of severe colitis, should be considered as potential direct or indirect complications of recent NSAID intake. Endoscopic studies are needed to determine whether cases of severe ambulatory NSAID induced diarrhoea are associated with lesions of colitis.

\section{ACKNOWLEDGEMENTS}

This study was supported by a grant awarded to IE by the Fondation pour la Recherche Médicale.

\section{Authors' affiliations}

I Etienney, Service de Gastroentérologie, Hôpital Rothschild, and Epidémiologie et Sciences de I'Information, INSERM Unit 444, Paris, France

L Beaugerie, Service de Gastroentérologie, Hôpital Rothschild, Paris, France

C Viboud, A Flahault, Epidémiologie et Sciences de l'Information, INSERM Unit 444, Paris, France

\section{REFERENCES}

1 Wolfe MM, Lichtenstein DR, Singh G. Gastrointestinal toxicity of non steroidal anti-inflammatory drugs. N Engl J Med 1999;340: 1888-99.

2 Biarnason I, Hayllar J, Macpherson AJ, et al. Side effects of non steroidal anti-inflammatory drugs on the small and large intestine in humans. Gastroenterology 1993;103:1832-47. 
3 Davies NM. Toxicity of non steroidal anti-inflammatory drugs in the large intestine. Dis Colon Rectum 1995;38:1311-21.

4 Morris AJ, Wasson LA, MacKenzie JF. Small bowel enteroscopy in undiagnosed gastrointestinal blood loss. Gut 1992;33:887-9.

5 Allison MC, Howatson AG, Torrance CJ, et al. Gastro-intestinal damage associated with the use of nonsteroidal anti-inflammatory drugs. N Engl J Med 1992;327:749-54.

6 Bjarnason I, Williams $\mathrm{P}$, So A, et al. Intestinal permeability and inflammation in rheumatoid arthritis: effect of non-steroidal anti-inflammatory drugs. Lancet 1998; ii: 1171-4.

7 Tibble JA, Sigthorson G, Foster R, et al. High prevalence of NSAID enteropathy as shown by a simple faecal test. Gut 1999:45:362-6.

8 Gibson GR, Withacre EB, Ricotti CA. Colitis induced by of nonsteroidal anti-inflammatory drugs: report of four cases and review of the literature. Arch Intern Med 1992;152:625-32.

9 Tanner AR, Raghunath AS. Colonic inflammation and nonsteroidal anti-inflammatory drug administration: an assessment of the frequency of the problem. Digestion 1988;41:116-20.

10 Glesson M, Ramsey D, Hutchinson S, et al. Colitis associated with non steroidal anti-inflammatory drugs. Lancet 1994;344:1028.

11 Colin R, Hochain P, Czernichow P, et al. Non steroidal anti-inflammatory drugs and segmental non-gangrenous colitis: a case-control study. Eur J Gastroenterol Hepatol 1993:5:715-19.

12 Evans JM, Mc Mahon AD, Mc Devitt DG, et al. Nonsteroidal anti-inflammatory drugs are associated with emergency admission to hospital for colitis due to inflammatory bowel disease. Gut 1997:40:619-22.

13 Riddell RH, Tanaka M, Mazzoleni G. Nonsteroidal anti-inflammatory drugs as a possible cause of collagenous colitis: a case-control study. Gut 1992;33:683-6.

14 Bohr J, Tysk C, Eriksson S, et al. Collagenous colitis: a retrospective study of clinical presentation and treatment in 163 patients. Gut 1996:39:846-51.

15 Beaugerie L, Berenbaum F, Berrebi $D$, et al. Chronic use of non-steroidal anti-inflammatory drugs does not alter colonic mucosa of patients without diarrhoea. Aliment Pharmacol Ther 2001;15:1301-6.

16 Hochberg MC. Diarrhea associated with nonsteroidal anti-inflammatory drugs. JAMA 1989;261:3081.
17 Colin C, Geffroy L, Maisonneuve H, et al. Country profile. France. Lancet 1997;349:791-7.

18 Letrilliart L, Desenclos JC, Flahault A. Risk factors for winter outbreak of acute diarrhoea in France: case-control study. BM 1997;315:1645-9.

19 Flahault A, Garnerin P, Chauvin P, et al. Sentinelle traces of an epidemic of acute gastroenteritis in France. Lancet 1995;346:162-3.

20 Maclure $\mathbf{M}$. The case-crossover design: a method for studying transient effects on the risk of acute events. Am J Epidemiol 1991;133:144-53.

21 Redelmeier DA, Tidshirdni RJ. Association between cellular telephone calls and motor vehicle collisions. N Engl J Med 1997;336:453-8.

22 Mittleman MA, Maclure M, Tofler GH, et al. Triggering of acute myocardial infarction by heavy physical exertion. Protection against triggering by regular exertion. Determinants of Myocardial Infarction Onset Study Investigators. N Engl J Med 1993;329:1677-83.

23 Mittleman MA, Maclure M, Tofler GH, et al. Triggering of acute myocardial infarction onset episodes of anger. Determinants of Myocardial Infarction Onset Study Investigators. Circulation 1995;92:1720-5

24 Mittleman MA, Maclure M, Nachnani M, et al. Educational attainment anger, and the risk of triggering myocardial infarction onset. Determinants of Myocardial Infarction Onset Study Investigators. Arch Intern Med 1997; 157:769-75.

25 Dixon KE. A comparison of case-crossover and case-control designs in a study of risk factors for hemorrhagic fever with renal syndrome. Epidemiology 1997;8:243-6.

26 Barbone F, McMahon AD, Davey PG, et al. Association of road-traffic accidents with benzodiazepine use. Lancet 1998:352:1331-6.

27 Confavreux C, Suissa S, Saddier P, et al. Vaccinations and the risk of relapse in multiple sclerosis. Vaccines in Multiple Sclerosis Study Group. NEngl J Med 2001;344:319-26.

28 Viboud C, Boëlle PY, Kelly J, et al. Comparison of case-control and case-crossover designs. J Clin Epidemiol 2001;54:1218-27.

29 Colombel JF, Dupas JL, Cortot A, et al. Incidence des maladies inflammatoires dans la région Nord-Pas-de-Calais et le département de la Somme (1988). Gastroenterol Clin Biol 1990;14:614-8.

30 Notteghem B, Salomez JL, Gower-Rousseau C, et al. Que deviennent les colites aiguës inclassées ? Résultats d'une étude de cohorte de 104 malades dans la région Nord-Pas-de-Calais. Gastroenterol Clin Biol $1993 ; 17: 811-5$

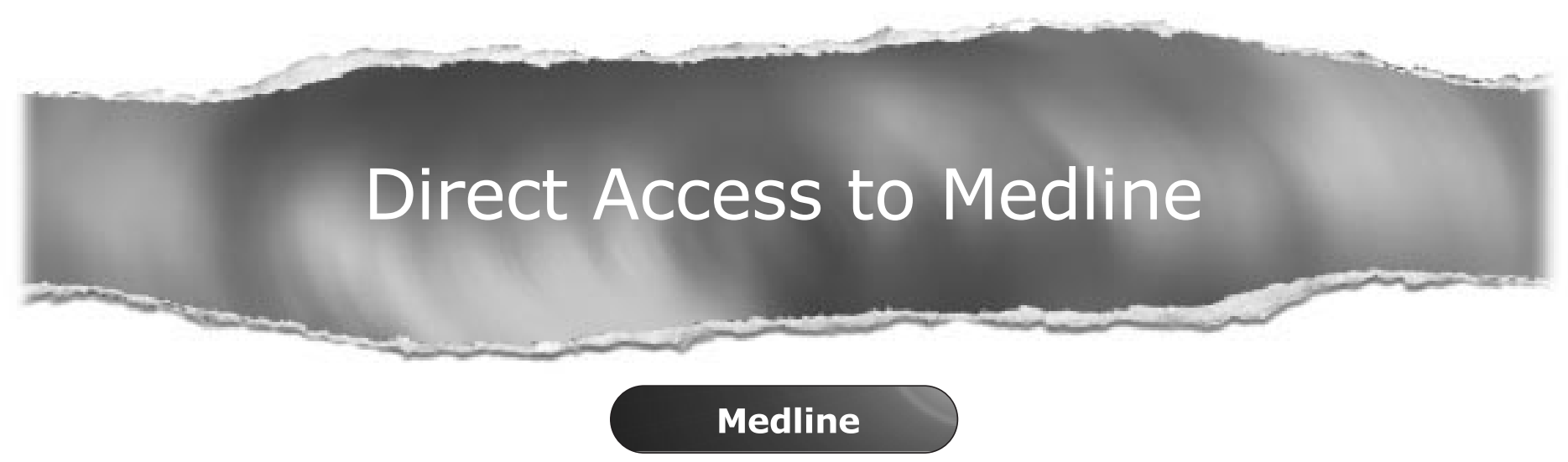

Link to Medline from the homepage and get straight into the National Library of Medicine's premier bibliographic database. Medline allows you to search across 9 million records of bibliographic citations and author abstracts from approximately 3,900 current biomedical journals. 